\title{
Projection methods based on grids for weakly singular integral equations
}

\author{
Filomena D. d' Almeida ${ }^{\mathrm{a}, *}$, Rosário Fernandes ${ }^{\mathrm{b}}$ \\ ${ }^{a}(C M U P)$ Centro de Matemática and Faculdade Engenharia da Universidade Porto, Rua \\ Roberto Frias, 4200-465 Porto, Portugal. \\ ${ }^{b}$ Centro de Matemática and Departamento de Matemática e Aplicações da Universidade do \\ Minho, Campus de Gualtar, 4710-057 Braga, Portugal.
}

\begin{abstract}
For the solution of a weakly singular Fredholm integral equation of the 2nd kind defined on a Banach space, for instance $L^{1}([a, b])$, the classical projection methods with the discretization of the approximating operator on a finite dimensional subspace usually use a basis of this subspace built with grids on $[a, b]$. This may require a large dimension of the subspace. One way to overcome this problem is to include more information in the approximating operator or to compose one classical method with one step of iterative refinement. This is the case of Kulkarni method or iterated Kantorovich method. Here we compare these methods in terms of accuracy and arithmetic workload. A theorem stating comparable error bounds for these methods, under very weak assumptions on the kernel, the solution and the space where the problem is set, is given.
\end{abstract}

Keywords: Projection approximations in $L^{1}$, weakly singular integral operators, error bounds.

2000 MSC: 65J10, 65R20.

\footnotetext{
* Corresponding author

URL: falmeida@fe.up.pt (Filomena D. d' Almeida), rosario@math.uminho.pt (Rosário Fernandes )
} 


\section{Introduction and notations}

Consider a weakly singular Fredholm integral equation of the 2nd kind

$$
T \varphi-z \varphi=f,
$$

where $z \neq 0$ is in the resolvent set of $T, f \in X$, and $T: X \rightarrow X$, is a compact linear integral operator on the space of Lebesgue integrable complex valued functions $X$, defined by

$$
(T \varphi)(\tau)=\int_{a}^{b} g\left(\left|\tau-\tau^{\prime}\right|\right) \varphi\left(\tau^{\prime}\right) d \tau^{\prime} .
$$

We can use classical projection methods where Eq. (1) is replaced by

$$
T_{n} \varphi_{n}-z \varphi_{n}=f,
$$

$T_{n}$ being a Galerkin, Sloan (iterated Galerkin) or Kantorovich as described by Atkinson in 8 .

Sloan in 15 had already described 4 variants of Galerkin methods based on orthogonal projections and Chatelin in $[9$ had described some of these methods in terms of non orthogonal projections, either for bounded and closed integral operators.

More recently, Kulkarni approximation and iterated Kantorovich approximation have been proposed (see [10, [11, [12, [5]). The Kulkarni approximation was presented for operators with smooth kernels, the iterated Kantorovich operator has been studied in [5] for the case where the kernel is weakly singular.

The Kulkarni method includes information about the initial operator $T$ that existes both in the Sloan (or iterated Galerkin Method) and Kantorovitch methods, so it approximates better $T$ but the arithmetic complexity of its computation is greater mainly when the basis in the approximating finite dimensional subspaces are not very rich.

In this work as we want to compare the methods of Kulkarni and iterated Kantorovich for weakly integral operators on Banach spaces, we are going to 
use the constants expressed in terms of the same quantities, namely the norm of the resolvent of the same operator, $T$.

Kulkarni method is very effective with a very good order of convergence with smooth kernels, smooth solution and very regular interpolatory projections. Under the conditions of this work, the error bounds given in Theorem 2, in terms of the same constants, show that the iterated Kantorovich method may be preferred as it is much easier to implement and have better error bounds.

As for notations we will use the superscripts $G, K, S, I K, R K$, respectively for Galerkin, Kantorovitch, Sloan, iterated Kantorovich and Kulkarni (Rekha Kulkarni) methods .

If we denote by $\left(\pi_{n}\right)_{n \geq 1}$ a sequence of bounded projections each one having finite-rank range $X_{n}$, we can represent the approximating operator $T_{n}$ by $T_{n}=$ $T_{n}^{G}:=\pi_{n} T \pi_{n}$ or $T_{n}=T_{n}^{K}:=\pi_{n} T$ or $T_{n}=T_{n}^{S}:=T \pi_{n}$, for classical Galerkin, Kantorovich and Sloan methods, respectively, and in the same manner by $T_{n}=$ $T_{n}^{R K}:=T_{n}^{K}+T_{n}^{S}-T_{n}^{G}=\pi_{n} T+T \pi_{n}-\pi_{n} T \pi_{n}$ for Kulkarni method. For the iterated Kantorovich method there is not a different operator, there is an improvement in the Kantorovich approximate solution thus yelding the iterated Kantorovich approximation $\varphi_{n}^{I K}:=\frac{1}{z}\left(T \varphi_{n}-f\right)$.

The assumptions made on $T$ ensure that Eq. (1) has a unique solution $\varphi \in X$, for any $f \in X$, that is $\varphi=R(z) f$, where $R(z):=(T-z I)^{-1}$ is the resolvent operator of $T$ (see [8]), and also that $T_{n}: X \rightarrow X$ is a bounded linear operator, $\left(T_{n}\right)_{n \geq 1}$ is $\nu$-convergent to $T$, in the sense that $\left(\left\|T_{n}\right\|\right)_{n \geq 1}$ is bounded, $\left\|\left(T_{n}-T\right) T\right\| \rightarrow 0$, and $\left\|\left(T_{n}-T\right) T_{n}\right\| \rightarrow 0$, and that $z \in \operatorname{re}\left(T_{n}\right)$, for $n$ large enough, as it can be seen in [6].

With that type of convergence of the approximating operators, the convergence of the approximate solution $\varphi_{n}$ to $T$ is guaranteed but a large value of $n$ may be needed. If the basis of the subspace $X_{n}$ is built on a grid on $[a, b]$ this leads to large linear systems to be solved as we will show in Section 2 One way to mitigate this disadvantage is to include more information in the approximating operator $T_{n}$, this is the motivation of Kulkarni method, or to compose one classical method with one step of iterative refinement, which is the 
case of iterated Kantorovich method. Here we compare two such methods with the classical ones in terms of accuracy and arithmetic workload.

This type of equations where the kernel of operator in (2) has a singularity along the diagonal (since it is given in terms of a singular function evaluated on the absolute value of the difference of two variables) plays a important role in several mathematical models.

It appears, for instance, in radiative transfer problems (we will take as an example a simplified model of radiative transfer in stellar atmospheres, interesting because the singular function is a multiple of the first exponential integral function [1]), in transient groundwater analysis, in image processing, probability theory, etc.

We consider that $g$ is weakly singular in the sense that $g:] 0,+\infty[\rightarrow \mathbb{R}$ is such that

$$
\begin{array}{r}
g\left(0^{+}\right)=+\infty, \\
g \in L^{1}([0,+\infty[) .
\end{array}
$$

We also add, with no considerable loss of generality, that $g \in C^{0}(] 0,+\infty[)$, $g \geq 0$ in $] 0,+\infty[$, and that $g$ is a decreasing function in $] 0,+\infty[$.

For the numerical solution of Eq. (3), the evaluation of a discretization matrix $A_{n}$, which represents the integral operator $T_{n}$ restricted to a finite dimensional space $X_{n}$, is required.

In the examples shown, kernel $g$ can be either the $g(s):=-\log (s / 2), s \in$ ]0,2] kernel (see [13]) or the radiative transfer in stellar atmospheres kernel, as described in [4] and [14]. $g(\tau):=\frac{\varpi}{2} E_{1}(\tau)=\frac{\varpi}{2} \int_{0}^{1} \frac{\exp (-\tau / \mu)}{\mu} d \mu, \tau>0$ $\left.a=0, b=\tau^{*}, \tau \in\left[0, \tau^{*}\right], \tau^{*} \in\right] 0,+\infty[$

The accuracy of the approximate solution depends, not only on the projection method used, but also on dimension of the discretization subspace, on the basis of this subspace, and on the accuracy of the evaluation of this discretization matrix.

In Section 2 we will present details of the computer implementation of these 
methods for weakly singular problems, and compare the arithmetic complexity of the algorithms.

In Section 3 we compare the iterated Kantorovich and Kulkarni methods, with the classical ones by producing theorems on their error bounds, and in Section 4 illustrations of performance of these in examples in $L^{1}([a, b])$ and $L^{2}([a, b])$ are given. Finally in Section 5 we draw some conclusions.

\section{Basis functions and discretization of the approximate operators}

When the basis functions of $X_{n}$ are defined by means of a grid on $[a, b]$ they may include the discontinuities of the problem as grid points.

In [2] the possible existence of boundary layers for the examples mentioned above, near the boundaries of the domain or where $f$ has a discontinuity is proved.

Let the basis en $=\left(e_{j}\right)_{j=1}^{n}$ for $X_{n}$ be made of the piecewise constant canonical functions when $X$ is the space of Lebesgue integrable functions,

$$
e_{j}(s):= \begin{cases}1 & \text { for } s \in\left[\tau_{j-1}, \tau_{j}\right] \\ 0 & \text { otherwise. }\end{cases}
$$

based on the grid $\mathcal{G}_{n}:=\left(\tau_{j}\right)_{j=0}^{n}$ such that $\tau_{0}:=a, \quad \tau_{n}:=b, \quad h_{j}:=\tau_{j}-\tau_{j-1}>$ 0 .

Its dual basis $e n^{*}$ is made of local mean functionals $e_{j}^{*}$ defined by

$$
\left\langle x, e_{j}^{*}\right\rangle:=\frac{1}{h_{j}} \int_{\tau_{j-1}}^{\tau_{j}} x(t) d t,
$$

and $\left\langle e n, e n^{*}\right\rangle:=I_{n}$, the idebtity matrix of order $n$.

The projection $\pi_{n}$ is then defined by $\pi_{n} x:=\sum_{j=1}^{n}\left\langle x, e_{j}^{*}\right\rangle e_{j} \quad$ for $x \in L^{1}([a, b])$.

For the classical Galerkin method the operator $T_{n}^{G}$, restricted to $X_{n}$ is represented by the matrix $A_{n}$ such that $A_{n}(i, j):=\left\langle T e_{j}, e_{i}^{*}\right\rangle$, and the equation (3) restricted to $X_{n}$ becomes

$$
\sum_{k=1}^{n} \sum_{j=1}^{n}\left\langle\varphi_{n}^{G}, e_{j}^{*}\right\rangle\left\langle T e_{j}, e_{k}^{*}\right\rangle e_{k}-z \varphi_{n}^{G}=\sum_{j=1}^{n}\left\langle f, e_{j}^{*}\right\rangle e_{j} .
$$


By applying $e_{i}^{*}$, for $i=1, \ldots, n$, we get

$$
\left(A_{n}-z I_{n}\right) x_{n}^{G}=f_{n},
$$

where $x_{n}^{G}(i):=\left\langle\varphi_{n}^{G}, e_{i}^{*}\right\rangle, f_{n}(i):=\left\langle f, e_{i}^{*}\right\rangle$.

After solving the linear system (4) the solution of (3) is recovered by the formula

$$
\varphi_{n}^{G}=\frac{1}{z} \sum_{j=1}^{n}\left(\left(A_{n} x_{n}^{G}\right)(j)-f_{n}(j)\right) e_{j}
$$

For the classical Kantorovich method the equation (3), $\left(T_{n}^{K}-z I\right) \varphi_{n}^{K}=f$, can be decomposed into its projection onto $X_{n}$ and onto $\left(I-\pi_{n}\right) X$ :

$$
\left\{\begin{aligned}
\left(\pi_{n} T-z \pi_{n}\right) \varphi_{n}^{K} & =\pi_{n} f, \\
-z\left(I-\pi_{n}\right) \varphi_{n}^{K} & =\left(I-\pi_{n}\right) f .
\end{aligned}\right.
$$

We can decompose correspondingly $\varphi_{n}^{K}=\varphi_{n, 1}^{K}+\varphi_{n, 2}^{K}$ and $f=f_{1}+f_{2}$, and obtain, after replacing the result of the second equation into the first,

$$
\left\{\begin{aligned}
T_{n}^{K} \varphi_{n, 1}^{K}-z \varphi_{n, 1}^{K} & =f_{1}+\frac{1}{z} T_{n}^{K} f_{2}, \\
\varphi_{n, 2}^{K} & =-\frac{1}{z} f_{2} .
\end{aligned}\right.
$$

On $X_{n}$, since $T_{n}^{K}=\pi_{n} T$,

$$
\sum_{j=1}^{n}\left\langle T \varphi_{n, 1}^{K}, e_{j}^{*}\right\rangle e_{j}-z \varphi_{n, 1}^{K}=f_{1}+\frac{1}{z} \sum_{j=1}^{n}\left\langle T f_{2}, e_{j}^{*}\right\rangle e_{j} .
$$

Applying $e_{i}^{*}$, for $i=1, \ldots, n$, and using

$$
\varphi_{n, 1}^{K}=\sum_{j=1}^{n}\left\langle\varphi_{n}^{K}, e_{j}^{*}\right\rangle e_{j}
$$


we get

$$
\left(A_{n}-z I_{n}\right) x_{n}^{K}=f_{n, 1}+\frac{1}{z} b_{n, 2},
$$

where $x_{n}^{K}(i):=\left\langle\varphi_{n, 1}^{K}, e_{i}^{*}\right\rangle, A_{n}(i, j):=\left\langle T e_{j}, e_{i}^{*}\right\rangle, f_{n, 1}(i):=\left\langle f_{1}, e_{i}^{*}\right\rangle, b_{n, 2}(i):=$ $\left\langle T f_{2}, e_{i}^{*}\right\rangle$ for $i, j=1, \ldots, n$.

After solving the linear system (5) the solution of (3) for the Kantorovich method is recovered by the formula

$$
\varphi_{n}^{K}=\varphi_{n, 1}^{K}+\varphi_{n, 2}^{K}=\sum_{j=1}^{n} x_{n}^{K}(j) e_{j}-\frac{1}{z} f_{2}
$$

For the Sloan method the equation (3), $\left(T_{n}^{S}-z I\right) \varphi_{n}^{S}=f$ means, since $T_{n}^{S}=T \pi_{n}$,

$$
\sum_{j=1}^{n} T\left\langle\varphi_{n}^{S}, e_{j}^{*}\right\rangle e_{j}-z \varphi_{n}^{S}=f
$$

or

$$
\sum_{j=1}^{n}\left\langle\varphi_{n}^{S}, e_{j}^{*}\right\rangle T e_{j}-z \varphi_{n}^{S}=f
$$

Applying $e_{i}^{*}$, for $i=1, \ldots, n$, we get

$$
\left(A_{n}-z I_{n}\right) x_{n}^{S}=f_{n}
$$

where $x_{n}^{S}(i):=\left\langle\varphi_{n}^{S}, e_{i}^{*}\right\rangle, A_{n}(i, j):=\left\langle T e_{j}, e_{i}^{*}\right\rangle, f_{n}(i):=\left\langle f, e_{i}^{*}\right\rangle$, for $i, j=1, \ldots, n$.

After solving system (6) the solution $\varphi_{n}^{S}$ is given by

$$
\varphi_{n}^{S}=\frac{1}{z} \sum_{j=1}^{n}\left(x_{n}^{S}(j) T e_{j}-f\right) .
$$

All these methods rely on the solution of a linear system of equations of dimension $n$ with the same coefficient matrix and differing only in the right hand side and the formula to recover the approximate solution $\varphi_{n}$.

The idea of the Kulkarni Method is to include in the operator $T_{n}^{R K}$ the information available in both the operators $T_{n}^{K}$ and $T_{n}^{S}$. 


$$
\begin{gathered}
\sum_{k=1}^{n} \sum_{j=1}^{n}\left\langle\varphi_{n}^{R K}, e_{j}^{*}\right\rangle\left\langle T e_{j}, e_{k}^{*}\right\rangle e_{k}+\frac{1}{z} \sum_{k=1}^{n} \sum_{j=1}^{n}\left\langle\varphi_{n}^{R K}, e_{j}^{*}\right\rangle\left\langle T T e_{j}, e_{k}^{*}\right\rangle e_{k}- \\
-\frac{1}{z} \sum_{t=1}^{n} \sum_{k=1}^{n} \sum_{j=1}^{n}\left\langle\varphi_{n}^{R K}, e_{j}^{*}\right\rangle\left\langle T e_{j}, e_{k}^{*}\right\rangle\left\langle T e_{k}, e_{t}^{*}\right\rangle e_{t}-z \sum_{j=1}^{n}\left\langle\varphi_{n}^{R K}, e_{j}^{*}\right\rangle e_{j}= \\
\sum_{j=1}^{n}\left\langle f, e_{j}^{*}\right\rangle e_{j}+\frac{1}{z} \sum_{j=1}^{n}\left\langle T f_{2}, e_{j}^{*}\right\rangle e_{j} .
\end{gathered}
$$

Applying $e_{i}^{*}$, for $i=1, \ldots, n$, we get

$$
\left(A_{n}+\frac{1}{z} B_{n}-\frac{1}{z} A_{n} A_{n}-z I_{n}\right) x_{n}^{R K}=f_{n}+\frac{1}{z} b_{n, 2},
$$

where

$$
x_{n}^{R K}(i):=\left\langle\varphi_{n}^{R K}, e_{i}^{*}\right\rangle, B_{n}(i, j):=\left\langle T T e_{j}, e_{i}^{*}\right\rangle i, j=1, \ldots, n .
$$

After solving system (7) the solution $\varphi_{n}^{R K}$ is given by

$$
\varphi_{n}^{R K}=\sum_{j=1}^{n} x_{n}^{R K}(j) e_{j}+\frac{1}{z}\left(\sum_{j=1}^{n} x_{n}^{R K}(j) T e_{j}-\sum_{j=1}^{n}\left(A_{n} x_{n}^{R K}\right)(j) e_{j}-f_{2}\right) .
$$

\section{Comparison in terms of arithmetic complexity}

For Kulkarni method the linear system to solve has the same dimension as the classical ones but the coefficient matrix is much more expensive in arithmetic operations, as it requires, besides $A_{n}$, the computation of a new matrix, $B_{n}$, representing the operator $T^{2}$ restricted to $X_{n}$ and the evaluation of $A_{n}^{2}$ which is very time consuming, if $n$ is large. Afterwards, the application of $T$ to $\sum_{j=1}^{n} x_{n}^{R K}(j) e_{j}$ or the pre-multiplication by a matrix representing $T$ on a much larger dimension subspace is required.

As for the iterated Kantorovich the approximation is obtained by using the Eq.11) to set a fixed point iteration and perform one step of this, starting with the approximation of Kantorovich, thus yielding

$$
\varphi_{n}^{I K}:=\frac{1}{z}\left(T \varphi_{n}^{K}-f\right)=\frac{1}{z}\left(T\left(\sum_{j=1}^{n} x_{n}^{K}(j) e_{j}-\frac{1}{z} f_{2}\right)-f\right) .
$$


The solution of a linear system with $A_{n}$ as coefficient matrix is needed to obtain $x_{n}^{K}$ and afterwords the application of $T$ to $\sum_{j=1}^{n} x_{n}^{K}(j) e_{j}$ or the premultiplication by a matrix representing $T$ on a much larger dimension subspace is required.

\section{Comparison in terms of accuracy}

The error bounds of the classical methods are summarized in the following theorem as proved in several results that can be seen, for instance in [6] .

Theorem 1. For $z \neq 0$, the projection approximations satisfy

$$
\begin{aligned}
& \frac{\left\|\varphi_{n}^{G}-\varphi\right\|}{\|\varphi\|} \leq C^{K}\left(\left\|\left(I-\pi_{n}\right) T\right\|+\frac{\left\|\left(I-\pi_{n}\right) f\right\|}{\|\varphi\|}\right), \\
& \frac{\left\|\varphi_{n}^{K}-\varphi\right\|}{\|\varphi\|} \leq C^{K}\left\|\left(I-\pi_{n}\right) T\right\|, \\
& \frac{\left\|\varphi_{n}^{S}-\varphi\right\|}{\|\varphi\|} \leq C^{S} \frac{1}{|z|}\|T\|\left(\left\|\left(I-\pi_{n}\right) T\right\|+\frac{\left\|\left(I-\pi_{n}\right) f\right\|}{\|\varphi\|}\right),
\end{aligned}
$$

for $n$ large enough, where

$$
C^{K}:=\sup _{n \geq n_{0}}\left\|\left(\pi_{n} T-z I\right)^{-1}\right\|, C^{S}:=\sup _{n \geq n_{0}}\left\|\left(T \pi_{n}-z I\right)^{-1}\right\| .
$$

In [2], 7] and [5] some relations between the basis and the error on the solutions are shown for the classical projection methods. If we want the constants to be comparable we can use $C^{K} \leq 2\left\|(T-z I)^{-1}\right\|, C^{S} \leq 2\left\|(T-z I)^{-1}\right\|$ (see [6]).

For the methods studied in this work we give the following theorem that compares the corresponding error bounds.

Theorem 2. For $n$ large enough, $z \neq 0$ in the resolvent set of $T$,

$$
\begin{aligned}
& \frac{\left\|\varphi_{n}^{I K}-\varphi\right\|}{\|\varphi\|} \leq 2 C\left\|\left(I-\pi_{n}\right) T\right\|\left\|\left(I-\pi_{n}\right) T^{*}\right\|, \\
& \frac{\left\|\varphi_{n}^{R K}-\varphi\right\|}{\|\varphi\|} \leq 2 C\left\|\left(I-\pi_{n}\right) T\right\|^{2}+\frac{\kappa}{|z|}\left\|\left(I-\pi_{n}\right) T\left(I-\pi_{n}\right)\right\|,
\end{aligned}
$$


where $C:=\left\|(T-z I)^{-1}\right\| /|z|$ depends on the norm of the resolvent operator, and

$\kappa:=\|T-z I\|\left\|(T-z I)^{-1}\right\|$ is the condition number of $T-z I$ relative to inversion.

Proof. Since

$$
\begin{aligned}
\varphi_{n}^{K}-\varphi & =\left(R_{n}^{K}(z)-R(z)\right) f \\
& =R(z)\left(T-T_{n}^{K}\right) R_{n}^{K}(z) f \\
& =R(z)\left(I-\pi_{n}\right) T \varphi_{n}^{K}
\end{aligned}
$$

we have

$$
\begin{aligned}
\varphi_{n}^{I K}-\varphi & =\frac{1}{z}\left(T \varphi_{n}^{K}-f\right)-\frac{1}{z}(T \varphi-f) \\
& =\frac{1}{z} T\left(\varphi_{n}^{K}-\varphi\right) \\
& =\frac{1}{z} R(z) T\left(I-\pi_{n}\right) T \varphi_{n}^{K} .
\end{aligned}
$$

Remarking that

$$
\begin{aligned}
\left\|T\left(I-\pi_{n}\right) T \varphi_{n}^{K}\right\| & =\sup \left\{\left|\left\langle T\left(I-\pi_{n}\right) T \varphi_{n}^{K}, x\right\rangle\right|: x \in X^{*},\|x\|=1\right\} \\
& =\sup \left\{\left|\left\langle\left(I-\pi_{n}\right) T \varphi_{n}^{K},\left(I-\pi_{n}^{*}\right) T^{*} x\right\rangle\right|: x \in X^{*},\|x\|=1\right\}
\end{aligned}
$$

for $n$ large enough, we have

$$
\begin{aligned}
\left\|T\left(I-\pi_{n}\right) T \varphi_{n}^{K}\right\| & \leq\left\|\left(I-\pi_{n}^{*}\right) T^{*}\right\|\left\|\left(I-\pi_{n}\right) T \varphi_{n}^{K}\right\| \\
& \leq 2\left\|\left(I-\pi_{n}\right) T\right\|\left\|\left(I-\pi_{n}^{*}\right) T^{*}\right\|\|\varphi\|,
\end{aligned}
$$

since $\left\|\varphi_{n}^{K}\right\| \leq 2\|\varphi\|$.

As for the Kulkarni approximation we have

$$
\begin{aligned}
\varphi_{n}^{R K}-\varphi & =\left(R_{n}^{R K}(z)-R(z)\right) f \\
& =R(z)\left(T-T_{n}^{R K}\right) R_{n}^{R K}(z) f \\
& =R(z)\left(T-\pi_{n} T-T \pi_{n}+\pi_{n} T \pi_{n}\right) R_{n}^{R K}(z) f \\
& =R(z)\left(\left(I-\pi_{n}\right) T-\left(I-\pi_{n}\right) T \pi_{n}\right) R_{n}^{R K}(z) f \\
& =R(z)\left(I-\pi_{n}\right) T\left(I-\pi_{n}\right) \varphi_{n}^{R K},
\end{aligned}
$$

and $\left(I-\pi_{n}\right) \varphi_{n}^{R K}=\left(\left(I-\pi_{n}\right) T \pi_{n} \varphi_{n}^{R K}-\left(I-\pi_{n}\right) f\right) / z$, thus yielding $\varphi_{n}^{R K}-\varphi=\frac{1}{z}\left(R(z)\left(I-\pi_{n}\right) T\left(I-\pi_{n}\right) T \pi_{n} \varphi_{n}^{R K}-R(z)\left(I-\pi_{n}\right) T\left(I-\pi_{n}\right) f\right)$. 
So, for $n$ large enough

$$
\begin{aligned}
\left\|\varphi_{n}^{R K}-\varphi\right\| \leq & \frac{1}{z}\left(\|R(z)\|\left\|\left(I-\pi_{n}\right) T\right\|^{2}\left\|\pi_{n}\right\|\left\|\varphi_{n}^{R K}\right\|+\right. \\
& \left.+\|R(z)\|\left\|\left(I-\pi_{n}\right) T\left(I-\pi_{n}\right) f\right\|\right) \\
\leq & \frac{1}{z}\left(2\|R(z)\|\left\|\left(I-\pi_{n}\right) T\right\|^{2}\left\|\pi_{n}\right\|\|\varphi\|+\right. \\
& \left.+\|R(z)\|\left\|\left(I-\pi_{n}\right) T\left(I-\pi_{n}\right)\right\|\|T-z I\|\|\varphi\|\right) \\
= & 2 C\left\|\left(I-\pi_{n}\right) T\right\|^{2}\|\varphi\|+\frac{\kappa}{|z|}\left\|\left(I-\pi_{n}\right) T\left(I-\pi_{n}\right)\right\|\|\varphi\|,
\end{aligned}
$$

where $\kappa:=\|T-z I\|\left\|(T-z I)^{-1}\right\|$ and $C:=\left\|(T-z I)^{-1}\right\| /|z|$, which leads to the second inequality, since $\left\|\pi_{n}\right\|=1$ in the framework described in Section 2 .

If we consider that $\left\|\left(I-\pi_{n}\right) T^{*}\right\|$ is of the order $\left\|\left(I-\pi_{n}\right) T\right\|$ the error bound for Kulkarni method has a extra term, $\frac{\kappa}{|z|}\left\|\left(I-\pi_{n}\right) T\left(I-\pi_{n}\right)\right\|$, when compared with the iterated Kantorovich method.

If these methods are used in Hilbert spaces, $H$, with self adjoint operators, the previous theorem can be simplified as follows, considering now the norm $\|\cdot\|_{H}$ induced by the inner product.

Theorem 3. If $T$ is selfadjoint, for $n$ large enough, and $z \neq 0$ in the resolvent set of $T$

$$
\begin{aligned}
\frac{\left\|\varphi_{n}^{I K}-\varphi\right\|_{H}}{\|\varphi\|_{H}} & \leq 2 C\left\|\left(I-\pi_{n}\right) T\right\|_{H}^{2} \\
\frac{\left\|\varphi_{n}^{R K}-\varphi\right\|_{H}}{\|\varphi\|_{H}} & \leq 2 C\left\|\left(I-\pi_{n}\right) T\right\|_{H}^{2}+\frac{\kappa}{|z|}\left\|\left(I-\pi_{n}\right) T\left(I-\pi_{n}\right)\right\|_{H} .
\end{aligned}
$$

\section{Examples}

The first example comes from a simplified model of radiative transfer in stellar atmospheres. It uses, as we mentioned, the first exponential integral function [1].

Equation (1) is set in the Banach space $X:=L^{1}\left(\left[0, \tau^{*}\right], \mathbb{C}\right)$. Its kernel is $g(s):=\frac{\varpi}{2} E_{1}(s)$, where $E_{1}$ is the first exponential integral function: 


$$
E_{1}(s):=\int_{0}^{1} \frac{\exp (-s / \mu)}{\mu} d \mu
$$

and $\left.s \in] 0, \tau^{*}\right]$ represents the optical depth of the stellar atmosphere and $\tau^{*} \in$ ] $0,+\infty[$ the optical thickness. The albedo $\varpi \in] 0,1[$ measures the scattering properties of the medium.

Here $z=1, \tau^{*}=100, \varpi=0.75$ and $f$ is defined by

$$
f(s):=\left\{\begin{array}{rll}
-1 & \text { for } \quad 0 \leq s \leq 50 \\
0 & \text { for } \quad 50<s \leq 100
\end{array}\right.
$$

\begin{tabular}{cccc}
\hline$n$ & Galerkin & Kantorovich & Iterated Kantorovich \\
\hline 500 & $1.2 \times 10^{-3}$ & $1.2 \times 10^{-3}$ & $1.2 \times 10^{-4}$ \\
1000 & $6.3 \times 10^{-4}$ & $6.3 \times 10^{-4}$ & $2.8 \times 10^{-5}$ \\
\hline
\end{tabular}

Table 1: $L^{1}$-norm relative errors

Table 1 shows the estimates of the relative errors in $L^{1}$-norm, with respect to a reference solution.

The grids on $[0,100]$ for this example are two uniform grids of 501 and 1001 points respectively. Computations have been performed with Matlab on a Intel Core $i 5-2410 M$ CPU @2.3 GHz, 4 GBytes of RAM DDR3.

We have computed the estimate relative error of the approximations with respect to a reference solution, obtained with a grid of 4001 points. The operator used to get this reference solution is used as a representation of $T$ in the Iterated Kantorovich method.

As we can see the Iterated Kantorovich method achieves a better error estimate than the classical methods.

The CPU times for this example, averaged over 10 runs of the methods, were respectively, following the order of Table 1, 76.65, 76.31 and 76.55 seconds for $n=500$, and 307.20, 306.08 and 306.46 seconds for $n=1000$. We did not add the time to build the large matrix representing the operator $\mathrm{T}$ (here we took it with dimension 4000) as it depends strongly on the examples and 
not on the methods under comparison. We took in account however, for the Iterated Kantorovich method, the time for the matrix-vector product with this large matrix that is needed independently of the example.

The Iterated Kantorovich method requires slightly more time than the Kantorovich method as it performs an extra matrix vector product as referred in Section 2,

As another example, we consider the function $g(s)=-\log (s / 2), s \in] 0,2]$, $z=4$ and the following right hand side function

$$
f(s):= \begin{cases}-1 & \text { if } 0 \leq s \leq 1 \\ 0 & \text { if } 1<s \leq 2 .\end{cases}
$$

In this example we will also compute the approximate solution with uniform grids of 501 and 1001 points, respectively. As we do not know the exact solution we will take as reference solution the one obtained with a uniform grid of 4001 nodes and use it in the computation of estimates of the relative errors corresponding to the two grids (see Table 2).

\begin{tabular}{cccc}
\hline$n$ & Galerkin & Kantorovich & Iterated Kantorovich \\
\hline 500 & $5.0 \times 10^{-4}$ & $5.0 \times 10^{-4}$ & $1.4 \times 10^{-6}$ \\
1000 & $2.5 \times 10^{-4}$ & $2.5 \times 10^{-4}$ & $3.3 \times 10^{-7}$ \\
\hline
\end{tabular}

Table 2: $L^{1}$-norm relative errors

In this case too, the Iterated Kantorovich method achieves a better error estimate than the classical methods.

The CPU times for this example, averaged over 10 runs of the methods, were respectively, $0.17,0.16$ and 0.18 seconds for $n=500$, and $0.49,0.48$ and 0.51 seconds for $n=1000$. Here again we did not add the time to build the large matrix representing the operator $\mathrm{T}$ (here we took it with dimension 4000).

Again the Iterated Kantorovich method requires more CPU time than the Kantorovich method. 
In the self adjoint case, we consider the space $H:=L^{2}([0,1], \mathbb{C})$ and the operator

$$
T x(s):=\int_{0}^{1} g(s, t) x(t) d t .
$$

with the kernel

$$
g(s, t):=10 \begin{cases}s(1-t) & \text { for } 0 \leq s \leq t \leq 1 \\ t(1-s) & \text { for } 0 \leq t \leq s \leq 1\end{cases}
$$

The source term $f$ is such that the exact solution is $\varphi(s):=s^{9 / 2}$.

In this case the basis is the set of $\left(P_{i}\right)_{i}$, where $P_{i}$ is the Legendre polynomial of degree $i, H_{n}:=\operatorname{Span}\left(P_{0}, P_{1}, \ldots, P_{n-1}\right), \pi_{n}$ is the orthogonal projection onto $H_{n}$.

\begin{tabular}{cccc}
\hline$n$ & Kantorovich & Kulkarni & Iterated Kantorovich \\
\hline 2 & $2.9 \times 10^{-1}$ & $1.4 \times 10^{+0}$ & $2.9 \times 10^{-1}$ \\
3 & $7.3 \times 10^{-2}$ & $1.7 \times 10^{-2}$ & $3.9 \times 10^{-2}$ \\
7 & $2.0 \times 10^{-5}$ & $2.0 \times 10^{-7}$ & $5.9 \times 10^{-7}$ \\
8 & $7.0 \times 10^{-7}$ & $3.0 \times 10^{-8}$ & $1.5 \times 10^{-8}$ \\
9 & $6.7 \times 10^{-8}$ & $6.1 \times 10^{-9}$ & $1.2 \times 10^{-9}$ \\
10 & $1.0 \times 10^{-8}$ & $1.5 \times 10^{-9}$ & $1.4 \times 10^{-10}$ \\
16 & $5.9 \times 10^{-12}$ & $3.6 \times 10^{-12}$ & $3.0 \times 10^{-14}$ \\
\hline
\end{tabular}

Table 3: $L^{2}$-norm relative errors

We compare the true relative errors in the $L^{2}-$ norm, as the exact solution is known. The iterations have been computed in Mathematica, Table 3 reports these results.

In this example we could use subspaces of smaller dimension than in the other examples because we could use orthogonal polynomials and the kernel has no singularities. Observing the behavior of one classical and the two new methods with increasing values of $n$, we can observe the evolution of the true relative errors. At first the Kulkarni method behaves better than the iterated 
Kantorovich but for $n$ larger than 7 the iterated Kantorovich is better. In general both the new methods are better than the classical Kantorovich method.

\begin{tabular}{ccc}
\hline Kantorovich & Kulkarni & Iterated Kantorovich \\
\hline 46.46 & 72.09 & 46.30 \\
\hline
\end{tabular}

Table 4: Average CPU times in seconds, for the third example, $n=16$

As for the CPU times in this example, Table 4 shows that the CPU time for the Rekha Kulkarni method is larger, as this method needs the computation of the $n \times n$ matrix $B_{n}$ as referred in Section 2. This takes 25.84 seconds approximately, which is almost $1 / 3$ of the CPU time for this method .

\section{Final remarks}

We compared two projection methods for weakly singular Fredholm integral equations in terms of accuracy and arithmetic complexity. These methods try and improve the classical ones by adding information about the operator to the iteration formula. Kulkarni method does this by building an approximate operator that includes the information of both Kantorovich and Sloan approximations. The iterated Kantorovich achieves that purpose by adding to the classical method one step of a fixed point iteration, using, in practice, a representation of the initial operator restricted to a larger dimension subspace, or , if possible the exact operator $T$. We showed that these are better in accuracy than the classical methods, by a theorem stating bounds for the relative error. Comparing Kulkarni method with the iterated Kantorovich, we conclude that the latter is faster since it has a smaller error bound, and the examples shown illustrate this behavior.

The projection methods rely on the solution of a linear system that has the same coefficient matrix for the classical methods. For Kulkarni method the linear system has a coefficient matrix that is much heavier in arithmetic computations, but on the other hand has better accuracy. Iterated Kantorovich 
method requires the computation of a representation of $T$ in a larger dimensional subspace, but only for one matrix vector product, and it has better accuracy too.

Acknowledgments. The authors warmly thank Mario Paul Ahues Blanchait for his collaboration in this work by useful remarks, suggestions and ideas.

The first author was partially supported by CMUP (UID/MAT/00144/2013), which is funded by FCT (Portugal) with national (MEC) and European structural funds (FEDER), under the partnership agreement PT2020.

\section{References}

[1] M. Abramowitz, I.A. Stegun, Handbook of Mathematical Functions, Dover, New York, 1960.

[2] M. Ahues, F. D. d'Almeida, R. Fernandes, Piecewise constant Galerkin Approximations of weakly singular integral equations, International Journal of Pure and Applied Mathematics, vol 55 (2009) 569-580.

[3] M. Ahues, F. D. d'Almeida, R. Fernandes, Error bounds for $L^{1}$ Galerkin approximations of weakly singular integral operators, Integral Methods in Science and Engineering, Vol. 2: Computational Methods, C. Constanda, M. E. Perez (Eds.) (2010) 1-10.

[4] M. Ahues, F. D. d'Almeida, A. Largillier, O. Titaud and P. Vasconcelos, An $L^{1}$ refined projection approximate solution of the radiation transfer equation in stellar atmospheres, J. Comput. Appl. Math 140 (2002) 13-26.

[5] M. Ahues, A. Amosov, A. Largillier, Superconvergence of some projection approximations for weakly singular integral equations using general grids, SIAM J. Numer. Anal. 47, No. 1 (2009) 646-674.

[6] M. Ahues, A. Largillier, B. V. Limaye, Spectral Computations with Bounded Operators, CRC, Boca Raton, 2001. 
[7] F. D. d'Almeida, M. Ahues, R. Fernandes, Errors and grids for projected weakly singular integral equations, International Journal of Pure and Applied Mathematics, vol 89 (2013) 203-213.

[8] K. Atkinson, The numerical solution of integral equations of the second kind, Number 4 in Cambridge Monographs on Applied and Computational Mathematics, Cambridge University Press, 1997.

[9] F. Chatelin, Spectral approximation of linear operators, Computer Science and Applied Mathematics, Academic Press, 1983.

[10] R. P. Kulkarni, A new superconvergent projection method for approximate solutions of eigenvalues problems, Numerical Functional Analysis and Optimization, Vol. 24, (1 and 2) (2003) 75-84.

[11] R. P. Kulkarni, A superconvergent result for solutions of compact operators equations, Bulletin of the Australian Mathematical Society, Vol. 68 (2003) $517-528$.

[12] R. P. Kulkarni, Approximate solution of multivariable integral equations of the second kind, Journal of Integral Equations and Applications, Vol. 16 (2004) 343-374.

[13] A. Pedas, Nyström type methods for a class of logarithmic singular Fredholm integral equations, Numerical Analysis and Applied Mathematics ICNAAM 2011, AIP Conf. Proc. 1389 (2011) 477-480.

[14] B. Rutily, Multiple scattering theory and integral equations, In Proceedings of Integral Methods in Science and Engineering (IMSE02), Birkäuser (2004) 211-232.

[15] I. H. Sloan, Four variants of the Galerkin method for integral equations of the second kind, IMA Journal of Numerical Analysis, Vol. 4 (1984) 9-17. 